\title{
Clinicomicrobial Profile of Neonatal Septicemia at a Tertiary Care Centre in Central India
}

\author{
Purti Tripathi $^{1}$ and Atul Rukadikar ${ }^{2 *}$ \\ ${ }^{1}$ Department of Microbiology, Government Medical College, Rajnandgaon, Chhattisgarh, India \\ ${ }^{2}$ Department of Microbiology, Chirayu Medical College, Bhopal, Madhya Pradesh, India \\ *Corresponding author
}

\section{A B S T R A C T}

Neonatal septicaemia is an important cause of morbidity and mortality among neonates constituting about $30-50 \%$ of total neonatal deaths in developing countries. Data from various studies reveal that results obtained vary from place to place and from period to period. Early diagnosis and proper management of neonatal septicemia can reduce the

\begin{tabular}{|l|}
\hline Key w or d s \\
Neonatal \\
septicaemia, \\
Antibiotic \\
susceptibility \\
testing.
\end{tabular}
morbidity and mortality substantially. 1) To study the bacterial etiologic agents responsible for neonatal sepsis. 2) To study the prevalent bacterial pathogens isolated from early onset neonatal sepsis (EOS) and late onset neonatal sepsis (LOS). 3) To study risk factors associated with neonatal sepsis. 4) To determine the susceptibility pattern of isolates to the commonly used antimicrobial agents in the treatment of sepsis. Blood culture reports were studied in 369 cases of clinically suspected septicemia in neonates using the standard conventional techniques. The antibiotic sensitivity was performed by Kirby-Bauer's disc diffusion method. In our study $66.93 \%$ had early onset sepsis and $33.06 \%$ had late onset sepsis. Male cases $(63.95 \%)$ outnumbered female cases $(36.04 \%)$. Premature rupture of membrane was the most common maternal risk factor (21.95\%), while low birth weight was most common neonatal risk factor $(70.73 \%)$.Blood culture reports were positive in $37.94 \%$ of cases. Klebsiella pneumonia and Enterococcus faecalis were the commonest organisms causing neonatal sepsis in EOS while Pseudomonas aeruginosa and Coagulase negative staphylococcus species in LOS. Continued surveillance of neonatal sepsis should be done in order to follow closely changes in trends and risk factors, to obtain information for empiric antibiotic therapy and to react rapidly in case of major changes in susceptibility patterns and occurrence of outbreaks.

\section{Introduction}

Neonatal septicemia is an important cause of morbidity and mortality among neonates constituting about $30-50 \%$ of total neonatal deaths in developing countries. ${ }^{(1)(2)}$ Data from National Neonatal Perinatal Database(NNPD, 2002-03) reveal that the incidence of neonatal sepsis is 30 per 1000 live births, ${ }^{(3)}$ sepsis develops in $20 \%$ neonates and $1 \%$ approximately die of sepsis related causes. ${ }^{(2)}$
Origin of Early onset sepsis (EOS) [within first week of life] is generally from pathogens of maternal genital tract, whereas late onset sepsis (LOS) [after first week till 28 days of life] is acquired either from the community or from hospital. ${ }^{(4)}$

Blood culture has a great diagnostic and prognostic significance in diagnosis of 
neonatal septicemia. But a positive blood culture does not necessarily confirm infection, since blood contamination can occur. ${ }^{(5)}$ If intermittent bacteremia occurs, multiple site culture may help improve pathogen detection. However, single site blood culture with blood volume of $>1 \mathrm{ml}$ can also detect neonatal sepsis. ${ }^{(6)}$

Data from various studies conducted over the last few decades reveal that results obtained vary from place to place and from period to period in which the studies were conducted. ${ }^{(7)}$ In the developing countries, E. coli, Klebsiella species and S. aureus being the most common pathogens of EOS, whereas S. aureus, Streptococcus pneumoniae, and Streptococcus pyogenes are the most commonly reported organisms in LOS. According to the National Neonatal perinatal Database of India, Klebsiella pneumoniae, Staphylococcus aureus and E. coli are the three most common organisms causing neonatal sepsis both in hospital and community. Moreover, the causative organisms of EOS and LOS sepsis are similar especially in hospital setting in developing countries. ${ }^{(8)}$ The present study was therefore conducted to determine the microbial etiology and the antimicrobial susceptibility of the microbial isolates for effective management of the cases.

\section{Materials and Methods}

The study was carried out over a period of one year at a tertiary care setting after getting approval from the institutional ethical committee. Neonates admitted with diagnosis of neonatal septicemia were included in study. As per the criteria by Vergnono et al., ${ }^{(7)}$ all the neonates presenting with convulsion, respiratory rate $>60$ breaths/min, severe chest indrawing, nasal flaring, grunting, bulging fontanel, redness around umbilicus extending to the skin, temperature $37.7^{\circ} \mathrm{C}$ or $<35.5^{\circ} \mathrm{C}$, lethargic or unconscious, reduced movements, not able to feed, crepitations, cyanosis, reduced digital capillary refill time $(>3$ seconds) were included in the study.

\section{Blood culture: ${ }^{(9)}$}

Two blood samples from each neonate, 1-2 ml of blood were collected by standard collection procedure using proper aseptic precautions and inoculated immediately into $20 \mathrm{ml}$ of brain heart infusion broth with $0.025 \%$ sodium polyanetholsulfonate as anticoagulant. Bottles were incubated at $37^{\circ} \mathrm{C}$ for 7 days. Three subcultures were made, first after $24 \mathrm{hr}$, and thereafter daily sub-culturing was done for 7 days on blood agar, MacConkey agar and chocolate agar. Identification of growth was by colony characters and standard biochemical tests.

Antibiotic sensitivity testing ${ }^{(9)}$ was performed by Modified Kirby-Bauer disc diffusion method ${ }^{(10)}$ as per CLSI recommendations. ${ }^{(11)}$ Control strains used were $S$. aureus ATCC 25923, E. coli ATCC 25922, P. aeruginosa ATCC 27853, E. faecalis ATCC 29212. Cefoxitin disc was used for testing methicillin resistance for Staphylococcal species. ${ }^{(11)}$ For detecting high level aminoglycoside resistance for Enterococcus spp., gentamicin $(120 \mu \mathrm{g})$ was used. ${ }^{(11)}$

All the gram negative isolates were further tested for ESBL productionusing CLSI phenotypic confirmatory test. ${ }^{(11)}$

\section{CLSI phenotypic confirmatory test}

ESBL was tested by applying the discs of ceftazidime and cefotaxime $(30 \mu \mathrm{g})$ and ceftazidime/ cefotaxime and clavulinic acid $(30 \mu \mathrm{g}+10 \mu \mathrm{g})$ to the lawn culture of the test organism. If the zone of inhibition around ceftazime/ ceftazidime clavulinic acid is $\geq 5$ $\mathrm{mm}$ than the zone of inhibition around 
ceftazidime disc, then the test organism is said to be ESBL producer.

Imipenem resistant Pseudomonas and Acinetobacter isolates were screened for metallo $-\beta$ lactamase production by combination disc test. ${ }^{(12)} \mathrm{A}$ colony of the suspected isolate was suspended in Mueller Hinton broth and turbidity was adjusted to 0.5 McFarland opacity standards. Lawn culture was prepared on Mueller Hinton agar and combination disc test was put. The combinations used were imipenem (I) and imipenem -EDTA (I-EDTA). Imipenem (10 $\mu \mathrm{g})$ and combined imipenem/EDTA $(750 \mathrm{mg})$ discs (Hi-media laboratories Pvt. Ltd., Mumbai) were placed on the agarplates. The test was considered MBL positive if a> $7 \mathrm{~mm}$ increase in the zone diameter for imipenem/EDTA was observed. ${ }^{(12)(13)}$

\section{Results and Discussion}

Total 369 neonates with neonatal septicemia were selected for present study. Early onset septicemia (0-72 hrs) was seen in 247 $(66.93 \%)$ cases and late onset septicemia in $122(33.06 \%)$ cases. Male preponderance was seen in both early onset AND late onset septicemia cases (Table 1). Among the maternal risk factors, premature rupture of membrane was seen in $81(21.95 \%)$ cases. The most common neonatal risk factor observed was low birth weight 261 (70.73\%), followed by prematurity $112(30.35 \%)$ (Table 2). Of the 247 early onset neonatal septicemia cases, blood culture was positive in $84(34 \%)$ cases. Similarly, out of 122 late onset neonatal septicemia cases, blood culture was positive in $56(45.90 \%)$ cases (Table 3 ).

Gram negative bacilli were found to be commonest cause of neonatal septicemia (68.57\%). Gram positive organisms were found in 39 (27.86\%) cases. Five Candida albicans were isolated (Table 4). Among 87 blood culture positive early onset neonatal septicemia cases, gram negative bacilli $(83.91 \%)$ were common aetiological agents as compared to gram positive cocci (16.09\%). Among gram negative bacilli, 29 Klebsiella pneumoniae, 17 Pseudomonas aeruginosa and 14 Acinetobacter baumannii were the common isolates. Among gram positive organisms Enterococcus fecalis (9 isolates) was the commonest organism (Table 4). In 53 blood culture positive late onset neonatal septicemia cases, gram negative bacilli isolated were $23(43.39 \%)$ and gram positive cocci were $25(47.17 \%)$ while Candida albicans were isolated in $5(9.44 \%)$ in number. Common bacterial isolates found in LOS were 18 coagulase negative Staphylococci followed by $9 P$. aeruginosa (Table 4).

Among gram negative isolates, all the isolates were found to be sensitive to imipenem except for 2 isolates of Acinetobacter and Pseudomonas each. Maximum resistance was seen to amoxyclav, ceftazidime, cefotaxime. Among aminoglycosides, gentamicin was found to be most resistant (Table 5). Among gram positive isolates, maximum sensitivity was for vancomycin and linezolid. Penicillin was found to be the most resistant (except for $S$. pneumoniae) followed by erythromycin and clindamycin (Table 6). ESBL production was detected in 40 isolates $(44.44 \%)$ by CLSI phenotypic method. Among these 40, 17 were Klebsiella spp., 12 Acinetobacter, 6 Pseudomonas and 5 E. coli. Two isolates (imipenem resistant) each of Pseudomonas aeruginosa and Acinetobacter baumannii were investigated for metallo $\beta$ lactamase (MBL) production. MBL was detected in one isolate each of Pseudomonas aeruginosa and Acinetobacter spp. MRSA was found in $3(37.5 \%)$ isolates and $5(25 \%)$ methicillin resistant CoNS. Three isolates (37.5\%) showed high level aminoglycoside resistance for Enterococcus ssp with gentamicin (120 
$\mu \mathrm{g})$ disc.

In this era of multidrug resistant organisms, neonatal septicemia remains an important challenging problem as it is associated with considerable morbidity and mortality. Identification of the microorganisms along with their antibiotic susceptibility pattern is one of the effective methods for proper management of neonatal septicemia cases.

Early onset septicemia (EOS) includes septicemia within less than $72 \mathrm{hrs}$ of birth and is associated with presence of perinatal risk factors, maternal genital tract being the source of infection. However, late onset septicemia (LOS) includes septicemia of more than 72 hrs of birth and source of infection is either nosocomial or community acquired. ${ }^{(14)}$

In our study, of the 369 neonatal septicemia cases, early onset septicemia (0-72 hrs) was seen in $247(66.93 \%)$ cases and late onset septicemia in $122(33.06 \%)$ cases. Chugh et al., ${ }^{(15)}$ had reported $68.8 \%$ cases of EOS and $32.2 \%$ cases of LOS. Movahedian et al., ${ }^{(16)}$ also reported a higher percentage of early onset septicemia $(77.5 \%)$ compared with late onset septicemia (22.5\%). In our study, 236 $(63.95 \%)$ male cases outnumbered 133 (36.04\%) female cases. The male to female ratio is $1.77: 1$ (Table 1). Our finding is comparable with Khatua et al., (17) who observed male to female ratio $1.7: 1$. Khatua et al., ${ }^{(17)}$ postulated that the factors regulating the synthesis of gamma globulins are probably situated on the $\mathrm{X}$ chromosome. Presence of one $\mathrm{X}$ chromosome in the male infant thus confers less immunological protection compared to female counterpart. Movahedian et al., ${ }^{(16)}$ also reported a higher male: female ratio $(2.64: 1)$.

Among the maternal risk factors, premature rupture of membrane was seen in 81 (21.95\%) cases (Table 2). Septicemia is more common among those infants whose mother had PROM with increased risk of contamination of amniotic fluid by organisms from birth canal before delivery. ${ }^{(18)}$ The most common neonatal risk factor observed was low birth weight $261(70.73 \%)$, followed by prematurity $112(30.35 \%)$ (Table 2). Khatua et al., ${ }^{(17)}$ reported LBW in $79.3 \%$ cases. Movahedian et al., ${ }^{(16)}$ reported $65 \%$ cases of $\mathrm{LBW}$ babies and $44.14 \%$ cases of prematurity.

Blood culture being the gold standard should be done in all suspected cases of sepsis prior to starting antibiotics. A positive blood culture and sensitivity of the isolate is the best guide to antimicrobial therapy. ${ }^{(19)}$ In the present study, blood culture positivity was seen in $37.94 \%$ and $62.06 \%$ were blood culture negative. Of the 247 early onset neonatal septicemia cases, blood culture was positive in 84 (34\%) cases. Similarly, out of 122 late onset neonatal septicemia cases, blood culture was positive in 56 (45.90\%) cases (Table 3). Murty et al., ${ }^{(5)}$ reported a blood culture positivity of $52.63 \%$.

Khatua et al., ${ }^{(17)}$ reported $59.80 \%$ of blood cultures as positive. Mathur et al., ${ }^{(20)}$ reported blood culture positivity of $24.88 \%$. In these studies and in the present study, there is a possibility of missing the anaerobes. In the present study, $62.06 \%$ blood cultures were negative and it does not rule out neonatal septicemia. These negative cultures may be due to antibiotic usage prior to sample collection, missed anaerobic infections. ${ }^{(5)}$

Gram negative bacilli were found to be commonest cause of neonatal septicemia $(68.57 \%)$ and $27.86 \%$ were gram positive organisms (Table 4). Khatua et al., ${ }^{(17)}$ reported $85 \%$ gram negative organisms and $15 \%$ gram positive organisms. Movahedian et al., ${ }^{(16)}$ also reported a higher percentage of gram negative organisms $(72.1 \%)$ as compared to $27.9 \%$ gram positive organisms. 
The increased susceptibility of neonates to the gram negative bacteria may be due to the fact that the antibodies against these organisms are mainly IgM type. It is not passively transferred through placenta and is present at very low level in blood at birth, reaching the adult level by the age of 2 years. Adequate IgG (except IgG 2-subtype) levels at term, afford protection against several gram positive bacteria. ${ }^{(21)}$

In blood culture positive early onset neonatal septicemia cases (87), gram negative bacilli (83.91\%) were common aetiological agents as compared to gram positive cocci (16.09\%).

Table.1 Age and sex distribution of neonatal septicemia cases $(n=369)$

\begin{tabular}{|l|c|c|c|}
\hline Age & Male (\%) & Female (\%) & Total (\%) \\
\hline $0-72$ hrs (Early onset septicemia) & $162(65.58)$ & $85(34.41)$ & $247(66.93)$ \\
\hline $72 \mathrm{hrs}-4$ wks (Late onset septicemia) & $74(60.65)$ & $48(39.34)$ & $122(33.06)$ \\
\hline Total & $236(63.95)$ & $133(36.04)$ & $369(100)$ \\
\hline
\end{tabular}

Table.2 Maternal and neonatal risk factors for neonatal septicemia $(n=369)$

\begin{tabular}{|l|c|}
\hline Risk factors & Number of septicemic neonates $(\%)$ \\
\hline Maternal & $81(21.95)$ \\
\hline $\begin{array}{l}\text { Premature rupture of membrane } \\
(\text { PROM) }>24 \mathrm{hrs}\end{array}$ & $66(17.88)$ \\
\hline $\begin{array}{l}\text { Febrile illness in mother in last 15 days } \\
\text { of delivery (Maternal fever) }\end{array}$ & \multicolumn{2}{|}{} \\
\hline Neonatal & $261(70.73)$ \\
\hline Low birth weight (weight $<2500 \mathrm{gm})$ & $112(30.35)$ \\
\hline Prematurity (gestational age $<37 \mathrm{wk})$ & $99(26.82)$ \\
\hline Neonatal resuscitation & $56(15.17)$ \\
\hline Lack of breast feeding & $47(12.73)$ \\
\hline $\begin{array}{l}\text { Superficial skin infection including } \\
\text { umbilical sepsis }\end{array}$ & $25(6.77)$ \\
\hline Meconium aspiration &
\end{tabular}

Table.3 Blood culture positivity in early onset and late onset septicemia $(n=369)$

\begin{tabular}{|l|c|c|c|}
\hline Blood Culture & EOS $(\boldsymbol{\%})$ & LOS $(\boldsymbol{\%})$ & Total $(\boldsymbol{\%})$ \\
\hline Positive & $84(34)$ & $56(45.90)$ & $140(37.94)$ \\
\hline Negative & $163(66)$ & $66(54.10)$ & $229(62.06)$ \\
\hline Total & $247(66.93)$ & $122(33.06)$ & $369(100)$ \\
\hline
\end{tabular}


Table.4 Microbial isolates from blood cultures of neonatal septicemia $(n=140)$

\begin{tabular}{|c|c|c|c|}
\hline Organisms & $\operatorname{EOS}(\%)$ & $\operatorname{LOS}(\%)$ & Total $(\%)$ \\
\hline Gram positive organisms & $14(16.09)$ & $25(47.17)$ & $39(27.86)$ \\
\hline Staphylococcus aureus & 02 & 06 & 08 \\
\hline $\begin{array}{l}\text { Coagulase negative } \\
\text { Staphylococci }\end{array}$ & 02 & 18 & 20 \\
\hline Enterococcus fecalis & 08 & 01 & 09 \\
\hline Streptococcus pneumonia & 02 & 00 & 02 \\
\hline Gram negative bacilli & $73(83.90)$ & 23 (43.39) & $96(68.57)$ \\
\hline E. coli & 07 & 03 & 10 \\
\hline Klebsiella spp. & 29 & 00 & 29 \\
\hline Citrobacter fruendii & 04 & 00 & 04 \\
\hline Enterobacter aerogens & 00 & 04 & 04 \\
\hline Salmonella typhi & 02 & 00 & 02 \\
\hline Acinetobacter spp. & 14 & 07 & 21 \\
\hline Pseudomonas aeruginosa & 17 & 09 & 26 \\
\hline Others & & $05(9.44)$ & $05(3.57)$ \\
\hline Candida albicans & 00 & 05 & 05 \\
\hline Total & $87(62.14)$ & $53(37.86)$ & $140(100)$ \\
\hline
\end{tabular}

Table.5 Antibiotic resistance pattern of gram negative isolates $(n=96)$

\begin{tabular}{|c|c|c|c|c|c|c|c|}
\hline Antibiotics & $\begin{array}{c}\text { E. coli } \\
(\mathrm{n}=10) \\
(\%)\end{array}$ & $\begin{array}{c}\text { Klebsiella } \\
\text { spp. }(\mathrm{n}=29) \\
(\%)\end{array}$ & $\begin{array}{c}\text { Citrobacter } \\
\text { fruendii }(\mathrm{n}=4)(\%)\end{array}$ & $\begin{array}{c}\text { Enterobacter } \\
\text { aerogens }(\mathrm{n}=4) \\
(\%)\end{array}$ & $\begin{array}{c}\text { S. typhi } \\
(\mathrm{n}=2) \\
(\%)\end{array}$ & $\begin{array}{c}\text { Acinetobacter spp. } \\
\qquad(\mathrm{n}=21) \\
(\%)\end{array}$ & $\begin{array}{c}\text { Pseudomonas } \\
\text { aeruginosa } \\
(\mathrm{n}=26)(\%)\end{array}$ \\
\hline Amoxyclav & $6(60)$ & $29(100)$ & $3(75)$ & $3(75)$ & - & - & - \\
\hline Piperacillin & $6(60)$ & $22(75.86)$ & $3(75)$ & $3(75)$ & - & $17(80.95)$ & $16(61.53)$ \\
\hline Ceftazidime & $7(70)$ & $26(89.65)$ & $3(75)$ & $2(50)$ & - & $16(76.19)$ & $18(69.23)$ \\
\hline Cefotaxime & $6(60)$ & $23(79.31)$ & $3(75)$ & $3(75)$ & $0(0)$ & $16(76.19)$ & $21(80.76)$ \\
\hline Cefepime & $0(0)$ & $10(34.48)$ & $3(75)$ & $1(25)$ & - & $16(76.19)$ & 19(73.07) \\
\hline $\begin{array}{l}\text { Piperacillin + } \\
\text { tazobactam }\end{array}$ & $1(10)$ & $0(0)$ & $1(25)$ & $0(0)$ & - & $5(23.8)$ & $9(34.61)$ \\
\hline Imipenem & $0(0)$ & $0(0)$ & $0(0)$ & $0(0)$ & - & $2(9.52)$ & $2(7.69)$ \\
\hline Gentamicin & $7(70)$ & $23(79.31)$ & $2(50)$ & $3(75)$ & - & $15(71.42)$ & $16(61.53)$ \\
\hline Amikacin & $1(10)$ & $15(51.72)$ & $2(50)$ & $2(50)$ & - & $10(47.61)$ & $10(38.46)$ \\
\hline Tobramycin & $4(40)$ & $19(65.51)$ & $1(25)$ & $1(25)$ & - & $15(71.42)$ & $16(61.53)$ \\
\hline Ciprofloxacin & $8(80)$ & $17(58.62)$ & $2(50)$ & $2(50)$ & $0(0)$ & $7(33.33)$ & $9(34.61)$ \\
\hline
\end{tabular}


Table.6 Antibiotic resistance pattern of gram positive isolates $(n=39)$

\begin{tabular}{|l|c|c|c|c|}
\hline Drugs & $\begin{array}{c}\text { Staph.aureus } \\
(\mathbf{n = 8})(\%)\end{array}$ & $\begin{array}{c}\text { Coagulase negative } \\
\text { Staphylococci } \\
(\mathbf{n = 2 0})(\%)\end{array}$ & $\begin{array}{c}\text { Enterococcus } \\
\text { faecalis }(\mathbf{n = 8})(\%)\end{array}$ & $\begin{array}{c}\text { Streptococcus } \\
\text { pneumoniae } \\
(\mathbf{n = 2})(\%)\end{array}$ \\
\hline Penicillin G & $8(100)$ & $20(100)$ & $8(100)$ & $0(0)$ \\
\hline Ampicillin & $6(75)$ & $15(75)$ & $5(62.5)$ & $1(50)$ \\
\hline Cefoxitin & $3(37.5)$ & $5(25)$ & - & $0(0)$ \\
\hline Erythromycin & $6(75)$ & $5(25)$ & $7(87.5)$ & $0(0)$ \\
\hline Clindamycin & $6(75)$ & $12(60)$ & - & - \\
\hline $\begin{array}{l}\text { Vancomycin } \\
\text { (E-test) }\end{array}$ & $0(0)$ & $0(0)$ & $0(0)$ & $0(0)$ \\
\hline Linezolid & $0(0)$ & $0(0)$ & $0(0)$ & $1(50)$ \\
\hline Gentamicin & $4(50)$ & $13(65)$ & $3(37.5)$ & $0(0)$ \\
\hline Amikacin & $5(62.5)$ & $14(70)$ & $3(37.5)$ & $1(50)$ \\
\hline Levofloxacin & $5(62.5)$ & $9(45)$ & $4(50)$ & - \\
\hline
\end{tabular}

Among gram negative bacilli, 29 Klebsiella pneumoniae, 17 Pseudomonas aeruginosa and 14 Acinetobacter baumannii were the common isolates. Among gram positive organisms Enterococcus fecalis (8 isolates) was the commonest organism (Table 4). In blood culture positive late onset neonatal septicemia cases (53), gram negative bacilli isolated were $23(43.39 \%)$ and gram positive cocci were $25(47.17 \%)$ while Candida albicans was isolated in $5(9.44 \%)$ cases. Common bacterial isolates found in LOS were 18 coagulase negative Staphylococci followed by $9 P$. aeruginosa (Table 4).

The bacteriological profile differs in EOS and LOS and it also differs in developing and developed countries. In developed countries gram positive organisms being predominant in both EOS and LOS, but Group B streptococci is more common in EOS. In developing countries gram negative organism are predominant in EOS and LOS both. $\left({ }^{22)}\right.$ Chugh et al., ${ }^{(15)}$ reported $90.31 \%$ gram negative bacilli in EOS and $29 \%$ positive organisms in LOS. Stoll et al., ${ }^{(2)}$ stated that majority of EOS $60.7 \%$ were caused by gram negative organisms. In a study by Guha et al., ${ }^{(23)} 1.6 \%$ S. aureus, $40 \%$ E. coli, $40 \%$
Klebsiella pneumoniae, 16.2\% Pseudomonas spp. from neonatal septicemia was reported. Mondal et al., (19) reported $15.2 \%$ Acinetobacter spp. isolates. Mathur et al., ${ }^{(20)}$ also reported Klebsiella pneumoniae (38.5\%) as the commonest organism isolated from neonatal septicemia, followed by $27.3 \%$ Enterobacter spp., 11.15 Pseudomonas spp., $6 \%$ E. coli, $10.25 \mathrm{~S}$. aureus, $2.1 \% \mathrm{~S}$. epidermidis, $0.9 \%$ Acinetobacter spp.. A study by Movahedian et al., ${ }^{(16)}$ reported Pseudomonas spp.(36\%) as the commonest organism isolated from neonatal septicemia, followed by $20.7 \%$ S. epidermidis, $17.1 \%$ Klebsiella spp., $10 \%$ Enterobacter spp., $6.3 \%$ Citrobacter spp., $6.3 \%$ S. aureus, $2.7 \%$ E. coli.

With advancing medical and surgical management strategies, nosocomial fungal infections are also on an increase, Candida spp. being the most common nosocomial fungal pathogen and $C$. albicans is the most commonly isolated species (24). We isolated $9.44 \%$ C. albicans in our study (Table 4). Rani et al., ${ }^{(24)}$ isolated $3.74 \%$ C. albicans. Arora et al., ${ }^{(25)}$ isolated $4.73 \%$ cases Candida spp. in their studies. The life threatening illness, varying microbiological etiology of 
neonatal septicemia and widespread antibiotic usage has resulted in antibiotic resistance. In our study, among gram negative isolates, all the isolates were found to be sensitive to imipenem except for 2 isolates of Acinetobacter baumannii and Pseudomonas aeruginosa each. Maximum resistance was seen to amoxyclav, ceftazidime, cefotaxime. Among aminoglycosides, gentamycin was found to be most resistant (Table 5).

Arora et al., ${ }^{(25)}$ had seen maximum resistance against ampicillin (89.96\%), cephalexin $(68.07 \%)$ and piperacillin $(57.71 \%)$. Movahedian et al., ${ }^{(16)}$ reports a very high degree of resistance among the gram negative organisms predominantly to broad spectrum cephalosporins. Gheibi et al., ${ }^{(14)}$ also reported high resistance to cefotaxime (67.5\%), ceftriaxone $(65.3 \%)$ and ceftazidime $(64.3 \%)$ among gram negative organisms but high sensitivity to amikacin (76.5\%) and ciprofloxacin $(92.8 \%)$.

Bhattacharjee et al., ${ }^{(26)}$ observed that more than $80 \%$ were sensitive to cefoperazone, amikacin, netilmicin, imipenem or piperacillin/tazobactam. Multidrug resistance was found in most of the gram negative bacilli strains in the present study.

Other workers have also reported multi drug resistance among the majority of gram negative isolates. ${ }^{(25)}$ Acinetobacter spp. has emerged as an multidrug resistant superbug challenging its clinical management, particularly in medical and surgical intensive care units. ${ }^{(27)}$ Bhattacharjee et al., ${ }^{(26)}$ reported that $P$. aeruginosa was sensitive to piperacillin/tazobactam (94\%), imipenem (86\%), cefoperazone (78\%), ceftazidime (79\%), ciprofloxacin (63\%), amikacin (68\%), gentamicin $(53 \%)$ and tobramycin $(38 \%)$.

Among gram positive isolates, maximum sensitivity was for vancomycin and linezolid.
Penicillin was found to be the most resistant (except for $S$. pneumoniae) followed by erythromycin and clindamycin (Table 6). Gheibi et al., ${ }^{(14)}$ also reported maximum susceptibility of gram positive isolates to vancomycin, ciprofloxacin. Arora et al., ${ }^{(25)}$ reported the resistance of gram positive organisms to ampicillin (74.61 \%) and erythromycin $(69.67 \%)$. Penicillin resistant stains, especially the hospital strains have increased to $75-90 \%$. Most penicillin resistant staphylococcal strains act by producing $\beta$ lactamases hydrolysing the $\beta$-lactam ring. ${ }^{(28)}$ Arora et al., ${ }^{(25)}$ reported Enterococcal drug resistance to ampicillin $(93.33 \%)$ and $86.67 \%$ resistance to erythromycin and linezolid.

Indiscriminate use of antibiotics has resulted in emergence of drug resistant strains. This has resulted in varying sensitivity pattern in different places and at times in same hospital at different times. Hence, it is very important to periodically review the strategies of antibiotic usage in neonates.

ESBL strains are associated with high mortality, being an important cause of nosocomial infection. ${ }^{(29)}$ ESBL production was detected in 40 isolates $(44.44 \%)$ by CLSI phenotypic method. Among these 40, 17 were Klebsiella spp., 12 Acinetobacter spp., 6 Pseudomonas spp. and 5 E. coli. Kim et al., ${ }^{(29)}$ reported ESBL production by $52.9 \%$ of $K$. pneumoniae and $17.9 \%$ of E. coli isolates.

Jain et al., ${ }^{(30)}$ have reported a high ESBL production by Klebsiella spp. (86.6\%), Enterobacterspp (73.4\%), E. coli $(65.3 \%)$ strains and in none of the isolates of Citrobacter spp. The high percentage of ESBL producing Klebsiella spp. may be due to selective pressure imposed by extensive use of antimicrobials. Intensive care unit, in which antibiotic use is heaviest and the potential for patient- to- patient transmission 
of organisms is greatest, is an important factor for ESBL production. ${ }^{(30)}$ In the present study, two isolates (imipenem resistant) each of Pseudomonas aeruginosa and Acinetobacter baumannii were investigated for metallo $\beta$ lactamase (MBL) production. MBL was detected in one isolate each of Pseudomonas aeruginosa and Acinetobacter spp. MRSA was found in $3(37.5 \%)$ isolates and $5(25 \%)$ methicillin resistant CoNS.

Three isolates (37.5\%) showed high level aminoglycoside resistance for Enterococcus ssp. with gentamicin $(120 \mu \mathrm{g})$ discs. Navneeth et al., ${ }^{(31)}$ reported that $12 \% P$. aeruginosa were MBL producers. Lee et al., ${ }^{(32)}$ had reported $15.1 \%$ MBL production in imipenem resistant Acinetobacter spp. There are limited studies from the Indian subcontinent on MBL production by imipenem resistant Acinetobacter spp. Further studies on MBL production should be carried out by Clinical Microbiology laboratories.

Neonatal septicemia is a life threatening emergency and the single most important cause of neonatal deaths in the community accounting for over half of them. A coordinated effort to limit inappropriate use of broad-spectrum antibiotics, efficient hospital antibiotic policies, vigilant detection of resistant species rigorous surveillance and infection-control protocols are needed to control the increasing incidence of highly drug resistant organisms.

Strict infection control in neonatal units, hand washing combined with judicious policy for antibiotic therapy are the main solution to this problem. Continued surveillance of neonatal sepsis should be done in order to follow closely changes in trends and risk factors, to obtain information for empiric antibiotic therapy and to react rapidly in case of major changes in susceptibility patterns and occurrence of outbreaks.

\section{References}

1. Bang AT, Bang RA, Baitule SB, Reddy $\mathrm{MH}$, Deshmukh MD. Effect of homebased neonatal care and management of sepsis on neonatal mortality: field trial in rural India. Lancet. 1999; 354(9194): 1955-61.

2. Stoll BJ. The global impact of neonatal infection. Clin Perinatol. 1997; 24: 121.

3. New Delhi: National Neonatology Forum NNPD Network. National Neonatology Forum NNPD Network National neonatal-perinatal database: report for 2002-2003. 2005;

4. Stoll BJ. Infections of the neonatal infant. In: Behrman RE, Kleigman RM $\mathrm{JH}$ (eds). $\mathrm{N}$ textbook of pediatrics. 17t. ed. PS 2004. Nelson textbook of pediatrics. 2004. 623-40 p.

5. Murty DS GM. Blood cultures in paediatric patients: A study of clinical impact. Indian J Med Microbiol. 2007; 25: 220-4.

6. Sarkar S, Bhagat I, DeCristofaro JD WT and SA. A study of the role of multiple site blood cultures in the evaluation of neonatal sepsis. J Perinatol. 2006; 26: 18-22.

7. Vergnono S, Sharland M, Kazembe P, Mwansambo HP. Neonatal sepsis: An international perspective. Arch Dis Child Fetal Neonatal Ed. 2005; 90: 220-4.

8. Sivanandan S, Soraisham AS SK. Choice and Duration of Antimicrobial Therapy for Neonatal Sepsis and Meningitis. Int J Pediatr. 2011;

9. Collee JG, Fraser AG, Marmion BP SA. Mackie and McCartney Practical Medical Microbiology. 14th ed. New York:Churchill-Livingstone. 1999.

10. Bauer AW, Kirby WM, Sherris JC TM. Antibiotic susceptibility testing by a 
standardized single disk method. Am J Clin Pathol. 1966; 45: 493-6.

11. Clinical and Laboratory Standard Institute 2016. Performance standard for antimicrobial susceptibility testing; twenty second informational supplement. CLSI document Jan 2016; M100-S Wayne, PA, USA. 2016.

12. Yong D, Lee K, Yum JH, Shin HB, Rossolini GB CY. Imipenem-EDTA disk method for differentiation of metallo- $\beta$-lactamase-producing clinical isolates of Pseudomonas spp. and Acinetobacter spp. J Clin Microbiol. 2002; 40: 3798-801.

13. Berges L, Rodriguez-Villalobos $\mathrm{H}$, Deplano A SM. Prospective evaluation ofimipenem/EDTA combined disc and Etest for detection of metallo-_lactamase-producing Pseudomonas aeruginosa. J Antimicrob Chemother. 2007; 59: 812--3.

14. Gheibi S, Fakoor Z, Karamyyar M, Khashabi J, Ilkhanizadeh B, Sana FA, Mahmoodzadeh H MH. Coagulase negative Staphylococcus; the most common cause of neonatal septicemia in Urmia, Iran. Iran J Pediatr. 2008; 18: 237-43.

15. Chugh K, Aggarwal BB KV and AS. Bacteriological profile of neonatal septicaemia. Indian J Pediatr. 1988; 55: 961-5.

16. Movahedian AH MR and MZ. Bacterial culture of Neonatal Sepsis. Iran J Publ Heal. 2006; 33: 84-9.

17. Khatua SP, Das AK, Chattergee BD, Khatua S GB et al., Neonatal septicaemia. Indian J Pediatr. 1986; 53: 509-14.

18. Saxena S, Anand NK SL and MS. Bacterial infections among home delivered neonates: Clinical picture and bacteriological profile. Indian Pediatr. 1980; 17: 17-24.
19. Mondal GP, Raghvan M BB and SS. Neonatal septicaemia among inborn and outborn babies in a referral hospital,. Indian J Pediatr. 1991; 58: 529-33.

20. Mathur M, Shah H, Dixit K, Khambadkone S CA. Bacteriological profile of neonatal septicemia cases (for the year 1990-91). J Postgr Med. 1994; 40: $18-20$.

21. Singh MB. publications; 2004. p.196216. Perinatal infections. In: Care of the newborn. 6th ed. New Delhi: Sagar. 2004. 196-216 p.

22. Ahmed ASMNU, Chowdhury MAKA, Hoque $M$ DG. Clinical and Bacteriological profile of neonatal septicemia in tertiary level pediatric hospital in Bangladesh. Indian Pediatr. 2002; 39: 1034-9.

23. Guha DK, Jaspal D, Das K, Khatri RL and SR. Outcome of neonatal septicemia- A clinical and bacteriological profile. Indian Pediatr. 1978; 15: 423-7.

24. Rani R, Mohpatra NP MG and RV. Changing trend of Candida species in a neonatal septicemia in a tertiary North Indian hospital. Indian J Med Microbiol. 2002; 20: 42-4.

25. Arora U and Devi P. Bacterial profile of blood stream infections and antibiotic resistance pattern of isolates. JK Sci. 2007; 9: 185-90.

26. Bhattacharjee A, Anupurba S GA and SM. Prevalence of inducible amp C $\beta$ lactamase- producing Pseudomonas aeruginosa in a tertiary care hospital in Northen India. IJMM. 2008;26:89-98.

27. Riley W. Acinetobacter and Moraxella. In: Borriello SP, Murray PR and Funke G Topley and Wilson's Microbiology and Microbial infections. Bacteriology Vol 2. 10th ed. Hodder Arnold publication; 2005. 1301-11 p.

28. Peacock SJ. Staphylococcus. In: Borriello SP, Murray PR and Funke G. 
Topley and Wilson's Microbiology and Microbial infections. Bacteriology Vol 2. 10th ed. Hodder Arnold publication ; 2005. 771-832 p.

29. Kim YK, Pari H, Lee HJ, Park SE CE. Bloodstream infections by Extended Spectrum $\quad \beta$-Lactamase-Producing Escherichia coli and Klebsiella pneumoniae in children: Epidemiology and clinical outcome. Antimicrob Agents Chemother. 2002; 46: 1481-91.

30. Jain A, Roy I, Gupta M KM and AS. Prevalence of Extended Spectrum $\beta$ Lactamase-Producing Gram-negative bacteria in septicemic neonates in a tertiary care hospital. J Med Microbiol. 2003; 52: 421-5.
31. Navneeth BV, Sridaran D, Sahay D BM. A preliminary study on metallo- $\beta$ lactamase producing Pseudomonas aeruginosa in hospitalised patient. Indian J Med Res. 2002; 116: 264-7.

32. Lee K, Ha GY, Shin BM, Kim JJ, Kang JO JS. Metallo-beta-lactamaseproducing Gram-negative bacilli in Korean Nationwide Surveillance of Antimicrobial Resistance group hospitals in 2003: Continued prevalence of VIM-producing Pseudomonas spp. and increase of IMP-producing Acinetobacter spp. Diagn Microbiol Infect Dis. 2004; 50: 51-8.

\section{How to cite this article:}

Purti Tripathi and Atul Rukadikar. 2017. Clinicomicrobial Profile of Neonatal Septicemia at a Tertiary Care Centre in Central India. Int.J.Curr.Microbiol.App.Sci. 6(12): 2036-2046. doi: https://doi.org/10.20546/ijcmas.2017.612.234 